\title{
RECONHECIMENTO DO PROFISSIONAL DE EDUCAÇÃO FÍSICA PELO CONSELHO NACIONAL DE SAÚDE: INTERVENÇÃO RECOMENDAÇÕES SOBRE CONDUTAS E PROCEDIMENTOS NA ATENÇÃO BÁSICA À SAÚDE
}

Cassio Hartmann ${ }^{1}$ Gabriel César Dias Lopes²

\section{Resumo}

O presente estudo teve como objetivo, apresentar para os profissionais de Educação Física que trabalham com a saúde coletiva, ás áreas e níveis de intervenção do profissional de Educação Física e as recomendações e condutas na Atenção Básica á Saúde. O Conselho Nacional de Saúde, reconhece o profissional da Educação Física sendo da área de saúde, desde 06 de março de 1997, através da Resolução no 218. De acordo com a Portaria 154/2008, as profissões que poderão compor os NASF (NÚCLEO DE SAÚDE DA FAMÍLIA) são: Médico, Acupunturista, Assistente Social, Profissional de Educação Física, Farmacêutico, Fisioterapeuta, Fonoaudiólogo, Médico Ginecologista, Médico Homeopata, Nutricionista, Médico Pediatra, Psicólogo, Médico Psiquiatra e Terapeuta Ocupacional. Este artigo justifica-se pela necessidade e carência de informações sobre a inserção do profissional de Educação Física na

\footnotetext{
${ }^{1}$ Professor de Educação Física - Delegado Adjunto Nacional da Federação Internacional de Educação Física. Mestrado em Ciência da Motricidade Humana. E-mail: cassiohartmann04@gmail.com
}

${ }^{2}$ Professor Orientador - E-mail: president@unilogos.education 
saúde coletiva. caracteriza-se por pesquisa qualitativa aquela que identifica fatores relevantes de um objeto de maneira a analisar o teor de documentos ou realidades, e ainda ressaltam a importância do embasamento teórico, assim sendo, trata-se de uma revisão bibliográfica acompanhada por uma pesquisa qualitativa aos documentos que regulamentam e legitimam a atuação do Profissional de Educação Física no sistema único de saúde e suas ações transversais. Pode-se concluir, que é de suma importância a intervenção do profissional de Educação Física no Programa Saúde da Família (PSF) e no NASF (NÚCLEO DE SAÚDE DA FAMÍLIA) nos três níveis de intervenção (primária, secundária e terciária), atuando na promoção da saúde e na prevenção de doenças, diretamente no tratamento não farmacológico e intervindo nos fatores de risco.

Palavras-chave: Saúde Coletiva; Atenção Básica á Saúde; Educação Física.

\section{Abstract}

The present study aimed to present to Physical Education professionals who work with collective health, the areas and levels of intervention of the Physical Education professional and the recommendations and conduct in Primary Health Care. The National Health Council recognizes the Physical Education professional being in the health area, since March 6, 1997, through Resolution №. 218. According to Ordinance 154/2008, the professions that may compose the NASF (FAMILY HEALTH CENTER) are: Doctor, Acupuncturist, Social Worker, Physical Education Professional, Pharmacist, Physiotherapist, Speech Therapist, Gynecologist, Homeopathic Doctor, Nutritionist, Pediatrician, Psychologist, Psychiatrist and Occupational Therapist. This article is justified by the need and lack of information about the insertion of the Physical Education professional in public health. Qualitative research is characterized by that which identifies relevant factors of an object in order to analyze the content of documents or realities, and still emphasize the importance of the theoretical basis, therefore, it is a bibliographic review accompanied by a qualitative research to documents that regulate and legitimize the performance of the Physical Education Professional in the single health system and its transversal actions. It can be concluded that the intervention of the Physical Education professional in the Family Health Program (PSF) and in the NASF (FAMILY HEALTH CENTER) is of paramount 
importance in the three intervention levels (primary, secondary and tertiary), working in the health promotion and disease prevention, directly in non-pharmacological treatment and intervening in risk factors.

Keywords: Collective Health, Primary Health Care, Physical Education.

\section{Resumen}

El presente estudio tuvo como objetivo presentar a los profesionales de Educación Física que trabajan con la salud colectiva, las áreas y niveles de intervención del profesional de Educación Física y las recomendaciones y conducta en Atención Primaria de Salud. El Consejo Nacional de Salud reconoce el profesional de Educación Física que se encuentra en el área de salud, desde el 6 de marzo de 1997, mediante la Resolución N²18. Según la Ordenanza 154/2008, las profesiones que pueden componer el NASF (CENTRO DE SALUD FAMILIAR) son: Doctor, Acupunturista, trabajador social, profesional de educación física, farmacéutico, fisioterapeuta, logopeda, ginecólogo, médico homeópata, nutricionista, pediatra, psicólogo, psiquiatra y terapeuta ocupacional. Este artículo está justificado por la necesidad y la falta de información sobre la inserción del profesional de Educación Física en salud pública. La investigación cualitativa se caracteriza por aquello que identifica los factores relevantes de un objeto para analizar el contenido de documentos o realidades, y aún enfatiza la importancia de la base teórica, por lo tanto, es una revisión bibliográfica acompañada de una investigación cualitativa para documentos que regulan y legitiman el desempeño del Profesional de Educación Física en el sistema único de salud y sus acciones transversales. Se puede concluir que la intervención del profesional de Educación Física en el Programa de Salud Familiar (PSF) y en el NASF (CENTRO DE SALUD FAMILIAR) es de suma importancia en los tres niveles de intervención (primaria, secundaria y terciaria), trabajando en el promoción de la salud y prevención de enfermedades, directamente en tratamientos no farmacológicos e interviniendo en factores de riesgo.

Palabras clave: Salud Colectiva; Atención Primaria de Salud; Educación Física. 


\section{INTRODUÇÃO}

O presente artigo justifica-se pela necessidade e carência de informações sobre a inserção do profissional de Educação Física na saúde coletiva. Objetivo geral desse estudo é apresentar ás áreas e níveis de intervenção do profissional de Educação Física e as recomendações e condutas na Atenção Básica á Saúde.

Desde o século XX a Organização Mundial da Saúde (WHO, 1958, p. 495) define saúde como "o perfeito bem-estar físico, mental e social do indivíduo, e não apenas a ausência de doenças e enfermidades", tornando mais amplo o conceito de saúde, percebendo que existem outros fatores a serem considerados além da inexistência de doenças. (AMERICAN HEART ASSOCIATION, 1992).

Para Mcardle (2002) a definição de saúde focaliza o amplo aspecto do bemestar que varia desde a ausência completa de saúde (morte) até os mais altos níveis de capacidade funcional.

Na Constituição Federal de 1988 no Brasil, refere-se, que a saúde é um direito de todos e um dever do estado, garantido mediante políticas sociais e econômicas que visem a redução do risco de doenças e agravos ao acesso universal e igualitário as ações e serviços para a sua promoção, proteção e recuperação.

O presente estudo tem como objetivo, apresentar para os profissionais de Educação Física que trabalham com a saúde coletiva, quais as categorias que se inserem de nível superior para fins de atuação do Conselho Nacional de Saúde e explanar as áreas de intervenção, recomendações sobre condutas e procedimentos na atenção básica á saúde, segundo o Conselho Federal de Educação Física (CONFEF, 2010).

O Conselho Nacional de Saúde, reconhece o profissional da Educação Física sendo da área de saúde, desde 06 de março de 1997, através da Resolução no 218.

Assim sendo houve a construção da integralidade da atenção a saúde, preceito constitucional do Sistema Único de Saúde (SUS), requer a atuação em equipes multiprofissionais e nesse sentido, a Educação Física é reconhecida como área de conhecimento e de intervenção acadêmico-profissional envolvida com a promoção prevenção, proteção e reabilitação da saúde.

Assim, o Conselho Nacional de Saúde - CNS - em Reunião Ordinária publica o seguinte texto: 
O Plenário do Conselho Nacional de Saúde em sua Octogésima Primeira Reunião Ordinária, realizada nos dias 07 e 08 de outubro de 1998, no uso de suas competências regimentais e atribuições conferidas pela Lei $n^{\circ} 8.080$, de 19 de setembro de 1990, e pela Lei $n^{\circ}$ 8.142, de 28 de dezembro de 1990, considerando que:

- a 8 Conferência Nacional de Saúde concebeu a saúde como "direito de todos e dever do Estado" e ampliou a compreensão da relação saúde/doença como decorrência das condições de vida e trabalho, bem como do acesso igualitário de todos aos serviços de promoção, proteção e recuperação da saúde, colocando como uma das questões fundamentais a integralidade da atenção à saúde e a participação social;

- a 10 CNS reafirmou a necessidade de consolidar o Sistema Único de Saúde, com todos os seus princípios e objetivos;

- a importância da ação interdisciplinar no âmbito da saúde;

- e o reconhecimento da imprescindibilidade das ações realizadas pelos diferentes profissionais de nível superior e estabelece um avanço no que tange à concepção de saúde e à integralidade da atenção.

\section{RESOLVE:}

I - Relacionar as seguintes categorias profissionais de saúde de nível superior para fins de atuação do Conselho:

1. Assistentes Sociais;

2. Biólogos;

3. Biomédicos;

4. Profissionais de Educação Física;

5. Enfermeiros;

6. Farmacêuticos;

7. Fisioterapeutas;

8. Fonoaudiólogos;

9. Médicos;

10. Médicos Veterinários;

11. Nutricionistas;

12. Odontólogos;

13. Psicólogos e

14. Terapeutas Ocupacionais.

II - Com referência aos itens 1, 2, 3 e 10, a caracterização como profissional de saúde deve ater-se a dispositivos legais e aos Conselhos de Classe dessas categorias. Resolução $\mathrm{n}^{0} \quad 287$ homologada pelo Presidente do Conselho Nacional de Saúde, José Serra no dia 08 de outubro de 1998, nos termos do Decreto de Delegação de Competência de 12 de novembro de 1991.

De acordo com a Portaria 154/2008, as profissões que poderão compor os NASF (NÚCLEO DE SAÚDE DA FAMÍLIA) são: Médico, Acupunturista, Assistente Social, Profissional de Educação Física, Farmacêutico, Fisioterapeuta, Fonoaudiólogo, Médico Ginecologista, Médico Homeopata, Nutricionista, Médico Pediatra, Psicólogo, Médico Psiquiatra e Terapeuta Ocupacional. Uma composição que reforça a importância do trabalho multiprofissional nas ações e programas relacionados a saúde. 
Iniciativas como os NASF tendem a mobilizar fortemente as diferentes categorias profissionais da saúde, além de provocar reflexões e estudos sobre as formas de atuação desses profissionais nesses campos específicos de intervenção.

A Política Nacional de Promoção da Saúde (BRASIL, 2006) conceitua as Práticas Corporais (atividades físicas) como expressões individuais e coletivas do movimento corporal, advindo do conhecimento e da experiência em torno do jogo, da dança, do esporte, da luta e da ginástica. São possibilidades de organização, escolhas nos modos de relacionar-se com o corpo e de movimentar-se, que sejam compreendidas como benéficas a saúde de indivíduos e de coletividades, incluindo caminhadas e práticas lúdicas, esportivas e terapêuticas.

Historicamente a Educação Física brasileira, esteve associada a qualidade de vida por meio da prevenção e manutenção da saúde e registra experiências bem sucedidas de atuação na área de saúde, em geral vinculadas a programas de instituições de ensino superior e hospitais universitários.

Essa associação resultou no reconhecimento por parte do Ministério da Saúde que instituiu no âmbito do Conselho Nacional de Saúde a Resolução nº 218, de 6 de março de 1997, a qual insere os profissionais de Educação Física na área da Saúde, e a Resolução no 287, de 8 de outubro de 1998 que relaciona a Educação Física entre as categorias profissionais de saúde de nível superior para fins de atuação do Conselho.

Neste contexto de desenvolvimento de recursos humanos na saúde, tem-se, ainda a institucionalização das Residências Multiprofissionais e em área Profissional da Saúde, em nível de pós-graduação lato sensu, além do Programa Nacional de Reorientação da Formação em Saúde - o Pro-Saúde e o Programa de Educação pelo Trabalho em Saúde - PET Saúde.

No estágio atual de desenvolvimento da Educação Física, a relação atividade física e saúde também avança, apoiada no estudo de pesquisadores da área e também na produção cientifica oriunda de áreas correlatas, sendo reconhecido o volume e a importância das pesquisas realizadas sobre o tema.

A configuração da Educação Física como uma profissão regulamentada, com seus respectivos direitos, deveres e as suas delimitações no âmbito das demais profissões da saúde. 
Segundo Marconi \& Lakatos (2017) caracterizam a pesquisa qualitativa aquela que identifica fatores relevantes de um objeto de maneira a analisar o teor de documentos ou realidades, e ainda ressaltam a importância do embasamento teórico, assim sendo, o presente estudo caracteriza como uma revisão bibliográfica acompanhada por uma pesquisa qualitativa aos documentos que regulamentam e legitimam a atuação do Profissional de Educação Física no sistema único de saúde e suas ações transversais.

\section{2 ÁREAS E NÍVEIS DE INTERVENÇÃO DO PROFISSIONAL DE EDUCAÇÃO FÍSICA}

De acordo com a Resolução nº 046/2002/CONFEF, que dispõe sobre a intervenção do profissional de Educação Física, essa define suas competências e campos de atuação profissional, a intervenção e plena nos serviços a sociedade no âmbito das atividades físicas, incluindo a prática de exercícios físicos e esportes, nas suas diversas manifestações e diferentes objetivos.

O profissional de Educação Física pode atuar como autônomo em instituições como em órgãos públicos e privados de prestação de serviços que envolvam a atividade física ou o exercício físico, incluindo aquelas responsáveis pela atenção básica a saúde, onde poderá atuar nos três níveis de intervenção (primária, secundária e terciária), dependendo das necessidades do indivíduo e do grau de competência do profissional.

Entende-se por intervenção primária, qualquer ato destinado a diminuir a incidência de uma doença numa população, reduzindo o risco de surgimento de casos novos.

A intervenção secundária busca diminuir a prevalência de uma doença numa população reduzindo sua evolução e duração, exigindo diagnóstico precoce e tratamento imediato.

A intervenção terciária visa diminuir a prevalência das incapacidades crônicas numa população, reduzindo ao mínimo as deficiências funcionais consecutivas a doença já existente, permitindo uma rápida e melhor reintegração do indivíduo na sociedade, com aproveitamento das capacidades remanescentes. 
O profissional de Educação Física, inserido na atenção básica á saúde deverá ser capaz de desenvolver ações compatíveis com as metas traçadas pelos órgãos responsáveis.

Este profissional atuará avaliando o estado funcional e morfológico dos beneficiários, estratificando e diagnosticando fatores de risco á saúde, prescrevendo, orientando e acompanhando exercícios físicos, tanto para pessoas consideradas "saudáveis", objetivando a promoção da saúde e a prevenção de doenças, quanto para grupos de portadores de doenças e agravos, atuando diretamente no tratamento não farmacológico e intervindo nos fatores de risco.

Cabe-Ihe, também, disseminar no indivíduo e na comunidade a importância da pratica de atividades físicas com base em conhecimentos científicos, desmistificando concepções equivocadas.

Atribui-se ao profissional de Educação Física as competências e habilidades para diagnosticar, planejar, organizar, supervisionar, coordenar, executar, dirigir, assessorar, dinamizar, programar, desenvolver, prescrever, orientar, avaliar, aplicar métodos e técnicas motoras diversas, aperfeiçoar, orientar e ministrar sessões específicas de exercícios físicos ou práticas corporais diversas (CONFEF 2002).

O profissional de Educação Física pode intervir no Programa Saúde da Família (PSF) tanto para orientar sobre a importância de hábitos de vida ativa, quanto para promover e estimular a adoção de um estilo de vida ativo, contribuindo para minimizar os riscos de doenças crônicas não transmissíveis e os agravos delas decorrentes.

Partindo desse pressuposto, cabe ao profissional de Educação Física, junto ao NASF e em outros espaços de intervenção, desenvolver ações que propiciem a melhoria da qualidade de vida da população, a redução dos agravos e danos decorrentes das doenças nao-transmissíveis, que favoreçam a redução do consumo de medicamentos, objetivando a prevenção e promoção da saúde por meio de práticas corporais, cabendo-Ihe, especificamente:

1) Proporcionar educação permanente por meio de ações próprias do seu campo de intervenção, juntamente com as Equipes de Saúde da Família (ESF), sob a forma de co-participação, acompanhamento e supervisão, discussão de casos e métodos da aprendizagem em serviço; 
2) Incentivar a criação de espaços de inclusão social, com ações que ampliem o sentimento de pertencimento social nas comunidades, por meio da atividade física regular, do esporte, das práticas corporais de qualquer natureza e do lazer ativo;

3) Promover ações ligadas aos exercícios/atividades físicas próprias do seu campo de intervenção junto aos orgãos públicos e na comunidade;

4) Articular parcerias com setores da área administrativa, junto com a ESF e a população, visando ao melhor uso dos espaços públicos existentes e a ampliação das áreas disponíveis para a prática de exercícios/atividades físicas próprias do seu campo de intervenção;

5) Promover eventos que estimulem e valorizem a pratica de exercícios/atividades físicas próprias do seu campo de intervenção, objetivando a saúde da população.

\subsection{Recomendações Sobre Condutas e Procedimentos do Profissional de Educação Física na Atenção Básica à Saúde}

As atividades ou exercícios físicos e práticas corporais devem ser desenvolvidas priorizando-se a inclusão de toda a comunidade, envolvendo não só as populações saudáveis, mas também aquelas com agravos manifestos da saúde ou mesmo em situação de maior vulnerabilidade.

Os profissionais de cada núcleo, em conjunto com a ESF e a comunidade, devem identificar as atividades, as ações e as práticas a serem adotadas com cada área contemplada no programa.

Considerando as exigências de qualidade para intervir na área da saúde, desenvolvendo programas de exercícios/atividades físicas próprias do seu campo de atuação, o profissional de Educação Física deve estar apto para as seguintes intervenções, dentre outras:

1) Aferir e interpretar os resultados de respostas fisiológicas durante o repouso e durante o exercício;

2) Coletar dados e interpretar informações relacionadas com prontidão para a atividade física, fatores de risco, qualidade de vida e nível de atividade física;

3) Aplicar escalas de percepção subjetiva do esforço; 
4) Manusear ergômetros (esteira, cicloergômetro, etc) e equipamentos utilizados em programas de exercício físico;

5) Manusear equipamentos usados para avaliação de parâmetros fisiológicos específicos;

6) Conhecer, aplicar e interpretar testes de laboratório e de campo utilizados em avaliação física;

7) Realizar testes de avaliação postural e de avaliação antropomêtrica;

8) Prescrever exercícios físicos baseados em testes de aptidão física, desempenho motor especifico, avaliação postural, índices antropométricos e na percepção subjetiva de esforço;

9) Trabalhar em equipe multiprofissional.

Para aplicação de avaliação física o profissional de Educação Física deve apresentar domínio de conhecimento em protocolos de testes e suas adequações de acordo com aptidão cardiorrespiratória do avaliado; indicações e contra-indicações para realização de testes; indicações de interrupção de testes; preparo de pacientes para a realização de testes; funcionamento de equipamentos; fisiologia do exercício e das respostas hemodinâmicas e respiratórias ao exercício físico; princípios e detalhes da avaliação, bem como os objetivos a serem atingidos.

Como todos os demais profissionais de uma equipe multidisciplinar de saúde, o profissional de Educação Física deverá conhecer a legislação específica da sua área de competência, para que não incorra em condutas e procedimentos que caracterizem práticas específicas de outras profissões da área da saúde.

Segundo (NUNES, 1998) há, portanto, a possibilidade de se estudar a Saúde Pública/Coletiva tentando periodizá-la, lembrando que as práticas que emergem nesses períodos não podem ser desarticuladas de inúmeros fatores, como também que certos objetos de estudo, como escreve Fourez (1995), somente têm condições de aparecer em dado momento histórico, enfatizando a construção cultural dos conceitos.

\section{CONSIDERAÇÕES FINAIS}

Pode-se concluir, que é de suma importância a intervenção do profissional de Educação Física no Programa Saúde da Família (PSF) e no NASF (NúCLEO DE 
SAÚDE DA FAMÍLIA) nos três níveis de intervenção (primária, secundária e terciária), atuando na promoção da saúde e na prevenção de doenças, diretamente no tratamento não farmacológico e intervindo nos fatores de risco.

Assim sendo o profissional de Educação Física, deve desenvolver ações que propiciem a melhoria da qualidade de vida da população, a fim de reduzir os agravos e danos decorrentes das doenças não-transmissíveis, que favoreçam a redução do consumo de medicamentos e principalmente trabalhar em equipe multidisciplinar e conhecer a legislação específica da sua área de competência, para que não incorra em condutas e procedimentos que caracterizem práticas específicas de outras profissões da área da saúde. 


\section{REFERÊNCIAS}

AMERICAN HEART ASSOCIATION. Statement on Exercise. Circulation, EUA, ํำ 86, p. 340-344, 1992.

BRASIL, MINISTÉRIO DA SAÚDE. Resolução CNS n‥ 218, de 6 de março de 1997. Reconhece as categorias profissionais consideradas como profissionais de saúde de nível superior. Diário Oficial da União. 5 maio 1997. Disponível em: http://sna.saude.gov.br/legisla/legisla/exerc_p/RES_CNS218_97exerc_p.doc, Acesso em: 15 de julho. 2019.

LAKATOS, Eva Maria. MARCONI, Marina de A. Fundamentos de Metodologia Científica. São Paulo: ebook, Atlas Editora, 2017.

McARDLE, William D.; KATCH, Frank I. \& KATCH, Victor L. Fisiologia do Exercício: Energia, Nutrição e Desempenho Humano. $3^{\circ}$ ed. Rio de Janeiro: GuanabaraKoogan, 1992.510 p.

NUNES, Everaldo Duarte. Saúde coletiva: história e paradigmas. Interface Comunic, Saúde, Educ. agosto 1998

RUIZ, João Álvaro. Metodologia científica: guia para eficiência nos estudos. 6. ed. São Paulo: Atlas, 2008.

SILVA, Francisco Martins da (organizador). Recomendações Sobre Condutas e Procedimentos do Profissional de Educação Física na Atenção Básica á Saúde. Rio de Janeiro. CONFEF, 2010.

STEINHILBER, Jorge. Resolução CONFEF no 46/2002. Dispões sobre a Intervenção do Profissional de Educação Física e respectivas competências e define os seus campos de atuação. Rio de Janeiro. CONFEF, 2002

WHO. The first ten years of the World Health Organization. Palais Nations, Geneue, Swtzeland. Constituition of the World Health Organition. 1958. 